\title{
Glanceable and Informative WearOS User Interface for Kids and Parents
}

\author{
Siyeon Kim ${ }^{1}$, Hyoseok Yoon ${ }^{1 *}$
}

\begin{abstract}
This paper proposes a wearable user interface intended for kids and parents using WearOS smartwatches. We first review what constitutes a kids smartwatch and then design UI components for watchfaces to be used by kids and parents. Different UI components ranging from activity, education, voice search, app usage, video, location, health, and quick dial are described. These components are either implemented as complications or on watchfaces and may require on-device standalone function, cross-device communication, and external database. We introduce a theme-based amusing UI for kids whereas simple and easily accessible components are recommended to parents' watchface. To illustrate use cases, we present 3 scenarios for enhancing communication between parents and child. To show feasibility and potential of our approach, we implement our proof-of-concept using commercial smartwatches, smartphones, and external cloud database. Furthermore, performance of checking app usages on different devices are presented, followed by discussion on limitations and future work.
\end{abstract}

Key Words: Smartwatch, user interface, wearable, WearOS.

\section{INTRODUCTION}

Smartwatch is one of the most successful commercial wearable devices that supports various use case scenarios by pairing with a main smartphone [1], [2], [3]. Without checking one's smartphone too often, smartwatches conveniently allow the wearers to find out current time and self-manage their schedule, activities, notification, messages, calls, and payments [4]. Commercial smartwatches currently on sale have different Operating Systems (OS), sensors, designs, and hardware buttons. Representative smartwatches include watchOS-based Apple Watch, Google's WearOS-based Android smartwatches, and Tizen-based Samsung smartwatches. On these platforms, programmers can develop cross-device applications (apps) for smartwatches and smartphones. Most smartwatches provide default apps related to personal health and activity management. However, default wearable user interfaces (UI) are not customized for different age groups of users and only provide limited use cases. For example, if we want to provide useful functions between parents and their kids, customized watchface and complications need to be designed and programmed separately. Particularly, current smart device (i.e., smartphone and smartwatch) usages can be identified to encourage recommended actions for reducing dependencies on the general-purpose smartphone to help kids concentrate. In this paper, we propose a wearable UI for immediately identifying the latest information of children and parents. The proposed wearable UI is programmed in WearOS by employing watchface and complications where the user can instantly check status of specific apps and execute frequently used functions even on the smartwatch's standby mode. Moreover, theme-based watchfaces and complications are designed, developed, and integrated to increase interests of intended children. On the other hand, parental watchface and complications are relatively simple and intuitive to easily perform functions such as launching video app, checking the child's current location, checking intake routine of medicine or nutritional supplements, and quick dialing to their kids.

\section{RELATED WORK}

\footnotetext{
Manuscript received February 26, 2021; Accepted March 26, 2021. (ID No. JMIS-21M-03-008)

Corresponding Author $\left(^{*}\right)$ : Hyoseok Yoon, 137 Hanshindae-gil, Osan-si, Gyeonggi-do, 18101, Republic of Korea, +82-31-379-0645, hyoon@hs.ac.kr.

${ }^{1}$ Division of Computer Engineering, Hanshin University, Osan-si, Gyeonggi-do, Korea, pennya6@hs.ac.kr, hyoon@hs.ac.kr
} 
A smartwatch is a wearable device whose usage varies with the user's lifestyle. By using a smartwatch, the wearer can manage their time efficiently without constantly checking on their smartphones. The notification filtering feature allows the user to receive only relevant information through immediate notifications. Smartwatches can also notify the wearer through vibration and audio feedback from the wrist in noisy and dynamic situations, so important notifications will not go unnoticed [5].

Smartwatches also free the hands of user eliminating the needs to hold any device. The wrist-worn feature eliminates the inconvenience of manually checking activity information such as elapsed workout time, calories burned, and current heart rate during intensive exercises such as jogging, biking, climbing, rafting, walking, and yoga. When the wearer's low activity level is detected with the built-in sensors on the smartwatch, stretching is recommended to them. There have been cases of emergency calls activated by the fall detection feature of Apple Watch. Overall, smartwatches have been actively used to manage the health of users in their daily lives as well as those who may be in critical situations.

Commercial smartwatches for kids are known as kids watches as shown in Fig. 1. These devices connect with parent's applications to send messages and receive child's location as well as their status. LG UPlus's Kakao Friends KidsWatch2 combined the popular characters of Kakao Friends to provide SOS button, protective settings, route monitoring and schedule management. MyFirst Fone S2 uses GPS to determine the location of a child in real time, issues an alarm on the parent's smartphone in case of emergency, and provides a step counter. VTech Kidizoom Smartwatch DX2 can be used to take videos, pictures, and selfies for customizing watch faces with filters.
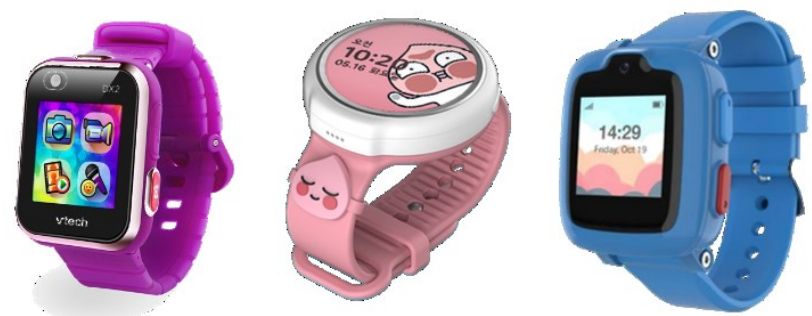

Fig. 1. Commercial kids watches (Vtech Kidizoom Smartwatch DX2, Kakao Friends KidsWatch 2, myFirst Fone S2).

\section{DESIGN OF GLANCEABLE AND INFORMATIVE USER INTERFACE}

Google's smartwatch operating system, WearOS, supports customized configuration by programming both the watchface and the complications shown in the watchface. This enables developers to design and implement specialized functions for various scenarios. "Glanceable interaction" refers to a type of interaction where the user can instantly identify, recognize, and react to the presented information. Google has applied this interactive principle to develop efficient Android apps.

We designed an interface for WearOS smartwatch with a focus on parents and children as intended smartwatch wearers. On the designed interface, we aim to provide frequently used functions and information such as location, app usage, educational games, activity information, and quick communication solution between parents and children. Most importantly, we wanted to relieve the anxiety of working parents who spend hours apart from their children.

\subsection{UI Components for Kids' Watchface}

The watchface for kids provides various components (i.e., complications or information presentation) for activity, education, voice search, and app usage as summarized in Table 1. We use more general term 'components' throughout this paper. Components in general can be implemented as complications in WearOS, but we also use non-complications such as non-interactive information display on a watchface. For kids, we also integrated a theme-based watchface and complications so they can be interested in the provided UI and amused to use the smartwatch regularly.

Table 1. Design considerations of WearOS components for kids.

\begin{tabular}{ll}
\hline Components & Brief Description \\
\hline Activity & Show personal activity level \\
Education & Check status of education app \\
Voice Search & Search using voice \\
App Usage & Daily app usage and time \\
\hline
\end{tabular}

We targeted our proposed WearOS UI to be used by elementary school students. They often use video apps, games, educational apps, music apps, and search functions. We considered four component types for kids. First type is activity component. This type of component is used to show the wearer's activity level. For example, a step counter can be placed to show the kid's number of steps.

Second type of component serves the role of education. We reserved one component slot to display current achievement level or used time of an education app. For example, a usage time for certain app can be displayed such as $25 \mathrm{~min}$ or level 3. This will work as a reminder for the kids to check their progress.

Third type of component is voice search. When this component is activated on the kid's smartwatch to accept voice input, the results of the voice search on the smartwatch can be confirmed through a smartphone. If the 
smartphone is far away, the voice search results can be directly played through the wireless earphone.

The fourth type of component is app usage. This component behaves like the second component type but can be applied for general apps. For example, app usage time for YouTube can be displayed in this component. This component is used to encourage children to use appropriate app for reasonable amount of time.

The child's watchface is designed as shown in Fig. 2 where useful information is visually expressed by using a background picture or a theme using characters.

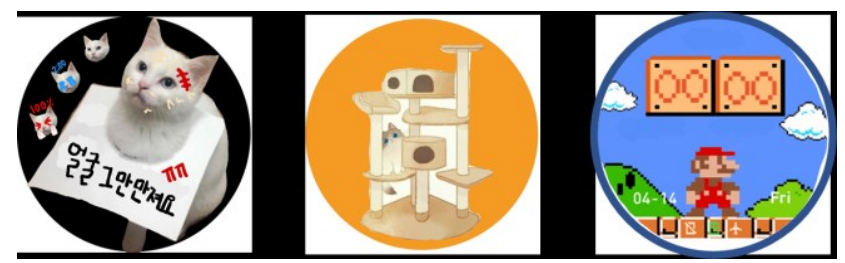

Fig. 2. Examples of theme-based watchfaces.

The left-most theme in Fig. 2 shows a cat whose appearances can be changed according to different states of used components. For example, if the number of face touches in a 'save face' complication reaches a warning level, the cat's face and messages change accordingly. The theme in center of Fig. 2 shows a cat tower where a cat can move between different tower locations. For example, if the wearer's activity level (i.e., step count) is too low, the cat moves to the top of the tower with a displayed message of "Let's take a walk". Different messages associated with different locations can be used with components. The rightmost theme of Fig. 2 shows a game character whose facial expressions or actions can be associated with different states of our complications.

Table 2. Examples of different component representations for kids.

\begin{tabular}{|c|c|c|c|c|}
\hline Component & Text & Icon & $\begin{array}{l}\text { Text + } \\
\text { Icon }\end{array}$ & Image \\
\hline Activity & face touch & $P$ & 85 & $x$ \\
\hline Education & $50 \%$ & & $\widehat{V}_{50 \%}$ & N/A \\
\hline Voice Search & voice search & Y & $\bigcup_{1}^{+}$ & N/A \\
\hline App Usage & $\begin{array}{r}5: 34 \\
\text { Youtuhe }\end{array}$ & $\infty$ & $\stackrel{5: 34}{\square}$ & N/A \\
\hline
\end{tabular}

The proposed WearOS UI for kids has a structure in which the above four components are integrated in. Table 2 shows possible combinations of component or complication types such as text only, icon only, text with icon, and images. Moreover, a dynamic or animated theme is exploited to amuse users as well as glanceable and informative to users. Fig. 3 shows our final design of kid's WearOS watchface. This design is revised as we implemented and tested on WearOS smartwatches due to a few technical issues, complexities, and aesthetics [6][7].



Fig. 3. Final design of kid's watchface and complications.

\subsection{UI Components for Parents' Watchface}

We designed watchface and components for parents focusing on increasing parental communication with their kids. In contrast to the kid's WearOS UI, the parents' WearOS UI is simple and intuitive. Therefore, other watchfaces and complications that satisfy the parents' needs can be used. From the perspective of parents with children, we considered components that either check status of their children or usable when they are together. Between parents and kids, video streaming services (i.e., YouTube and Netflix), location services, children's health status, and phone call are frequently used functions. Therefore, we chose four components to cover video, location, health, and quick dial as summarized in Table 3.

Table 3. Design considerations of WearOS components for parents.

\begin{tabular}{ll}
\hline Component & Brief Description \\
\hline Video & $\begin{array}{l}\text { Launches YouTube with registered keywords } \\
\text { on the parent's smartphone }\end{array}$ \\
$\begin{array}{l}\text { Location } \\
\text { Displays current location of the child }\end{array}$ \\
$\begin{array}{l}\text { Checks/Un-checks nutritional supplement or } \\
\text { medicine in-take }\end{array}$ \\
\hline
\end{tabular}

Video component is a function showing playing a video with the registered keywords and applications in the associated smartphone. For example, when a keyword "car" has been pre-registered, clicking this component launches YouTube app on the parent's smartphone with a list of search results.

Location component allows the parents to instantly see 
their child's location. More specifically, the child's location can be categorized into home, school, and academy. It is a feature to show the child's current location, so a location update module should be installed and approved on the child's smartwatch or smartphone in advance.

For health component, we designed a feature to quick check or uncheck intake of medicines and nutritional supplements such as Vitamin C. This complication does not require integration with their child's smartwatch. It simply works as a toggle to remind and indicate whether daily medicine or nutritional supplements are given or not.

Quick dial complications allow the parents to instantly call the phone number of the registered child. If you have multiple children, multiple quick dial complications can be selected on the watchface.

\subsection{Scenarios}

We illustrate how our proposed wearable UIs for parents and kids may be used in three scenarios depicted in Fig. 4.

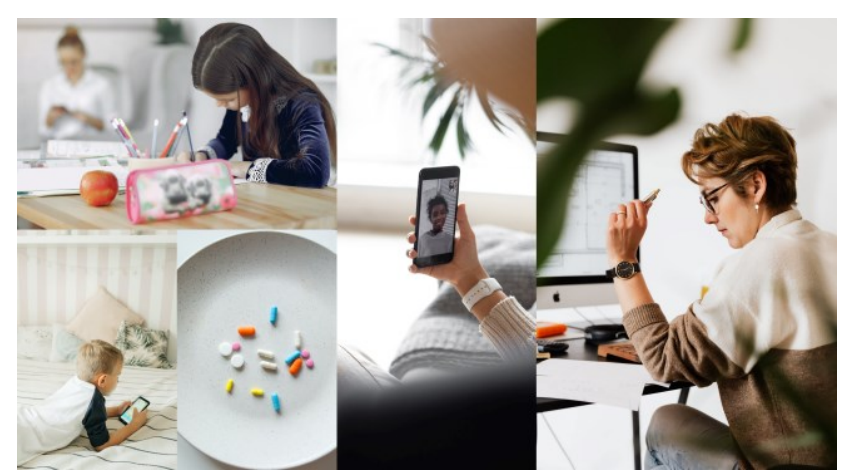

Fig. 4. Scenarios for the proposed wearable UI.

Scenario \#1: Parents have gone to work. The child's daily schedule routine is in an order of school, academy, and home. From time to time, parents check whereabouts of her by looking at the watchface. When she arrives at home, a quick dial on the watchface is used to call the child.

Scenario \#2: The child is having difficulties with arithmetic. He has made a promise to work on his arithmetic skills by playing an education app on the smartphone for 30 minutes regularly. His playing time is displayed on the watchface and his favorite hero character smiles when he reaches 30 minutes of the playing time.

Scenario \#3: The child is taking two Vitamin-C pills daily. To remind the child and parents, a toggle complication on their watchfaces is used to indicate whether the child has taken the pills today. When the parent checks his watchface, he realizes that the health complication is grayed out. The parent gives the pills to his child and clicks on the health complication after the in-take. Now he sees on his watchface that a green 'OK' is displayed on the health complication.

\section{IMPLEMENTATION OF WEAROS UI}

We implemented our own versions of several components described in Section 3 in WearOS to demonstrate feasibility of the proposed user interface. WearOS shares identical development environment with Android, so WearOS works easily with Android-based smartphones to launch apps or share data. In addition, external clouds or databases can be used to share information collected from smartphones/smartwatches for sharing between parents and children. Fig. 5 shows the data sharing structure used in the watchface.

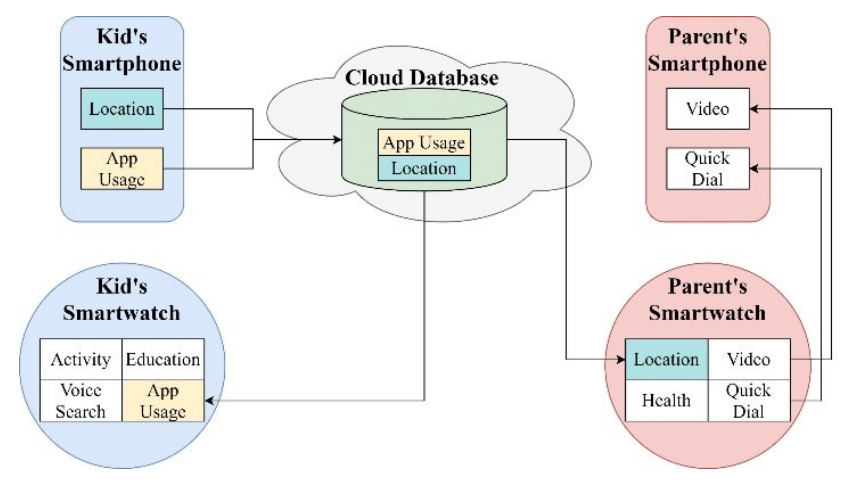

Fig. 5. Data sharing structure of the proposed wearable UI.

Among UI components, Activity and Education are stand-alone components that can be performed on-device (i.e., on a smartwatch). Components such as Voice Search, Video, and Quick Dial can be implemented as Intents so that a simple trigger from the smartwatch can execute a specific app or function on the smartphone. The usage time of a specific app and the usage time of an educational game are implemented by acquiring the usage time information using UsageStatsManager with INTERVAL_DAILY to go through installed package names on the smartphone and the smartwatch. The usage time collected from the smartphone is periodically saved on a cloud database which can be periodically or retrieved on-demand by the watchface and complications. Also, the child's location information is generated from the child's smartphone GPS and saved to an external cloud or database. This location information is retrieved periodically or on-demand by the parent's watchface and complications.

Table 4. Specification of two commercial smartwatches.

\begin{tabular}{llll}
\hline Specification & \multicolumn{2}{l}{ Fossil Q Explorist } & \multicolumn{2}{l}{ Xiaomi Mi Watch } \\
\hline WearOS & v2.24 & v2.23 & \\
Processor & Snapdragon Wear & Snapdragon & Wear \\
& 2100 & & 3100 \\
\\
RAM/Storage & $512 \mathrm{MB} / 4 \mathrm{~GB}$ & $1 \mathrm{~GB} / 8 \mathrm{~GB}$ & \\
\hline
\end{tabular}


We implemented on one relatively old and one recent commercial WearOS smartwatches as shown in Table 4. Fig. 6 shows our implemented proof-of-concept (PoC) WearOS UI.

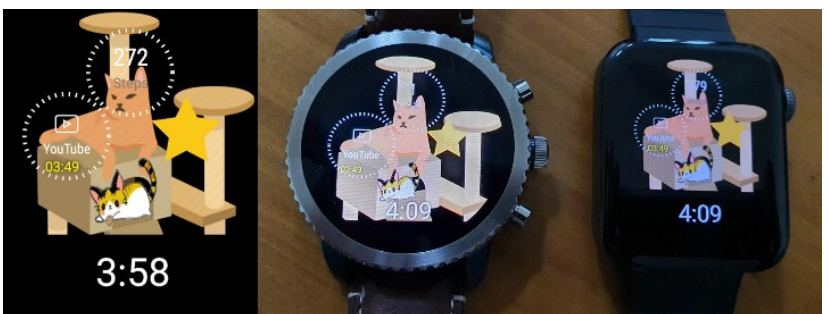

Fig. 6. Proof-of-concept WearOS UI implementation, screen capture, running on Fossil Q Explorist and Xiaomi Mi Watch.

This PoC is an example of WearOS UI for kids. The watchface has a cat tower-based theme where animated cat is displayed on the bottom component. On the top complication slot, a general complication from WearOS, step counter is placed. On the left, YouTube complication is shown as icon with text type. The usage time of YouTube is augmented separately to the complication as yellow text, since the complication in WearOS has strict specification and technical limitations (i.e., icon color, external database connection). On the right component, a filled star is displayed when an education app usage is sufficient.

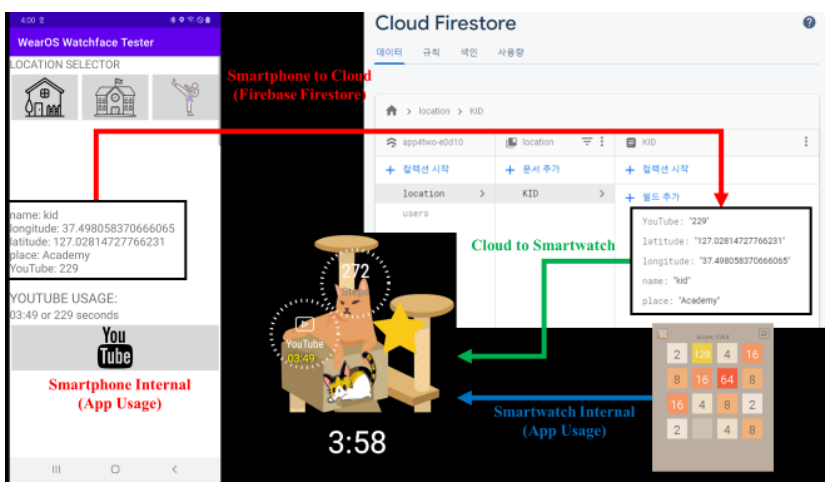

Fig. 7. Data sharing among smartphone, smartwatch, and cloud.

Fig. 7 shows how different devices and cloud database are connected to share data. We used a companion smartphone app (WearOS Watchface Tester) to simulate a child's smartphone to send location information and smartphone app usage (i.e., YouTube) to an external cloud database. For the database, we used Firebase Firestore. If a component can work stand-alone or all information can be gathered on-device, no external connection is needed. However, if some information is on the smartphone (i.e., smartphone app usage) or on the child's smartphone (i.e., location information), then the intermediary cloud database is used.

\section{EXPERIMENT RESULTS}

Our proposed wearable UI has two possible performance bottlenecks. First one is when we connect to an external cloud database to send and retrieve data such as app usage time and location information. Second one is when we internally check installed packages to find a target app and calculating the usage time of that app. Since the first bottleneck depends on network conditions, we examined the second bottleneck with two commercial smartwatches and smartphones for on-device performance of using UsageStatsManager. As described earlier, to find a usage time of an app, we need to go through the installed packages until we find the target app. We measured the time to check all the installed packages on different devices. Table 5 shows the experiment results. We found that performance of using UsageStatsManager in commercial smartwatches and smartphones ranged from $737 \mathrm{~ms}$ to $3,375 \mathrm{~ms}$ with the installed packages numbers from 82 to 1,318 . This is reasonable since we just need to update usage time of an app periodically such as 30 minutes or 1 hour interval.

Table 5. UsageStatsManager performance and \# of packages

\begin{tabular}{lll}
\hline Specification & UsageStatsManager & Packages \\
\hline Fossil Q Explorist & $1,159 \mathrm{~ms}$ & 82 \\
Xiaomi Mi Watch & $737 \mathrm{~ms}$ & 167 \\
Samsung A31 & $3,375 \mathrm{~ms}$ & 712 \\
Samsung Note 10 & $1,718 \mathrm{~ms}$ & 1318 \\
\hline
\end{tabular}

\section{CONCLUSIONS}

In this paper, we proposed a WearOS UI using watchface and components of several functions that can be used between parents and children. To design the WearOS UI, we considered characteristics and needs of the child and parents. Our aim was to enhance communication between parents and children through simple yet interesting and accessible interface of the smartwatch. We demonstrated feasibility of our approach through illustrated scenarios as well as by assessing performance of implemented PoC on commercial smartwatches and smartphones.

This work is not without limitations. The strict specification of WearOS watchface and complication conflicted with our initial design where we wanted to present visually expressive and colorful complications and sharing data externally. To deal with this technical limitation, we used an external cloud database to communicate with the watchface module instead of directly communicating with the complications. Also, this work deals with sensitive data of children and parents, therefore follow-up studies on strengthening security and data 
protection are desired [8]. Moreover, we only considered visual components in our work such as changing icons, text, and characters. Other modalities such as vibration and sound [5] are interesting future work to consider along with usability and user experience evaluation on specialized tasks. Lastly, we targeted only two specific groups (parents and children) in our work. Based on our WearOS UI, we can expand our work to more diverse groups such as for seniors, students, and teachers.

\section{Acknowledgement}

This work was supported by the Basic Science Research Program through the National Research Foundation of Korea (NRF) funded by the Ministry of Education (NRF-2018R1D1A1B07043983). Photos used in Fig. 4 are all from Pexels with the free license to use (https://www.pexels.com/license).

\section{REFERENCES}

[1] C. Saatjohann, F. Ising, L. Krings, and S. Schinzel, "STALK: Security analysis of smartwatches for kids," in Proceedings of the $15^{\text {th }}$ International Conference on Availability, Reliability and Security, Virtual Event Ireland, Article 25, Aug. 2020.

[2] A. Tavakoulnia, K. Guzman, F. L. Cibrian, K. D. Lakes, G. Hayes, and S. E. B. Schuck, "Designing a wearable technology application for enhancing executive functioning skills in children with ADHD," in Adjunct Proceedings of the 2019 ACM International Joint Conference on Pervasive and Ubiquitous Computing and Proceedings of the 2019 ACM International Symposium on Wearable Computers, London, United Kingdom, pp. 222-225, Sep. 2019.

[3] S. A. Pérez, A. M. Díaz, and D. M. López, "Personalized tracking of physical activity in children using a wearable heart rate monitor," International Journal of Environmental Research and Public Health, vol. 17, no. 16, Article 5895, Aug. 2020.

[4] P. Santoso, F. Pasila, and I. H. Putro, "Mobile sensing platform using smartwatch," in Proceedings of 2018 Electrical Power, Electronics, Communications, Controls and Informatics Seminar, Batu, East Batu, East Java, Indonesia, pp. 430-433, Oct. 2018.

[5] H. Yoon and J. Son, "Collocated wearable interaction for audio book application on smartwatch and hearables," Journal of Multimedia Information System, vol. 7, no. 2, pp. 107-114, Jun. 2020.

[6] W. Jackson, SmartWatch Design Fundamentals. Berkeley, CA: Apress, 2019.

[7] Wear OS by Google - Introduction, https://designguidelines.withgoogle.com/wearos/, 2020.
[8] M. Williams, J. R. C. Nurse, and S. Creese, "Smartwatch games: encouraging privacy-protective behavior in a longitudinal study," Computers in Human Behavior, vol. 99, pp. 38-54, Oct. 2019.

Authors



Siyeon Kim is an undergraduate student in the Division of Computer Engineering at Hanshin University. In May 2020, she joined the HCI Lab at Hanshin University as an undergraduate researcher. Her research interests include assistive technology, wearable computing, and Human-Computer Interaction.

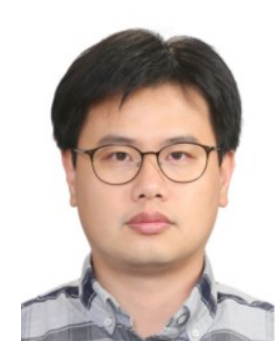

Hyoseok Yoon received his B.S. degree in Computer Science from Soongsil University in 2005. He received his M.S. and Ph.D. degrees in Information and Communication (Computer Science and Engineering) from the Gwangju Institute of Science and Technology (GIST), in 2007 and 2012, respectively. He was a researcher at the GIST Culture Technology Institute from 2012 to 2013 and was a research associate at the Korea Advanced Institute of Science and Technology (KAIST), Culture Technology Research Institute in 2014. He was a senior researcher at Korea Electronics Technology Institute (KETI) from 2014 to 2019. In September 2019, he joined the Division of Computer Engineering, Hanshin University where he is currently an assistant professor. His research interests include ubiquitous computing (context-awareness, wearable computing) and Human-Computer Interaction (mobile and wearable UI/UX, MR/AR/VR interaction). 